\title{
Análisis de la influencia de la modalidad deportiva sobre la victimización en escolares de Primaria
}

(E) Analysis of the influence of the sports modality on victimization in primary school children

\author{
Zurita Ortega, Felix ${ }^{1}$; Castro Sánchez, Manuel²; Chacón Cuberos, Ramon ${ }^{3}$
}

\section{Resumen}

Introducción: La violencia y su erradicación es un elemento que se debe de considerar de vital importancia en la educación, puesto que acontecimientos de bullying se producen cada vez más a una temprana edad. La actividad física constituye uno de los principales elementos para el trabajo de prevención.

Objetivos: Por lo que se plantea este estudio con los objetivos de describir los niveles de victimización, actividad física y tipo de deporte en una población de 9 a 12 años de la ciudad de Granada y establecer la relación entre la práctica y tipología de actividad física y los niveles de victimización.

Métodos: Participaron 177 escolares de 10 a 12 años $(\mathrm{M}=11,04$ años; $\mathrm{DT}=1,258)$ de la ciudad de Granada a los que se les aplicaron un cuestionario Ad-Hoc y el cuestionario de victimización en la escuela.

Resultados y discusión: Los resultados depararon que tres cuartos de los escolares realizan actividad física no escolar, siendo esta más asidua entre los varones, y estos se caracterizaban por tener un índice superior de victimización física mientras que el género femenino se caracteriza por tener mayor victimización relacional, es decir tienden más a excluir a los compañeros y aunque esta sea menos visible puede provocar grandes daños psicológicos. Las modalidades deportivas favoritas entre los alumnos activos son los deportes individuales sin contacto en las chicas y el deporte colectivo con contacto entre los varones, las conductas violentas eran más frecuentes en los deportes colectivos además de que los alumnos practicantes se exponían a situaciones de victimización manifiesta verbal y física en la mayoría de situaciones

Palabras clave: Victimización; Educación Primaria; Deporte; Escolares.

\section{Abstract}

Introduction: Violence and its eradication is an element that should be considered of vital importance in education, since bullying events occur more and more at an early age. Physical activity is one of the main elements for prevention work.

Aim: For this study is proposed with the objectives of describing the levels of victimization, physical activity and type of sport in a population of 9 to 12 years of the city of Granada and establish the relationship between the practice and type of physical activity and the levels of victimization

Methods: A total of 177 school children from 10 to 12 years old $(\mathrm{M}=11.04$ years old, $\mathrm{SD}=1.258)$ from the city of Granada participated in an Ad-Hoc questionnaire and the victimization questionnaire in the school.

Results and discussion: The results showed that three quarters of the students perform non-school physical activity, this being more frequent among males, and these were characterized by having a higher rate of physical victimization while the female gender is characterized by having greater victimization relational, that is, they tend more to exclude peers and although this is less visible it can cause great psychological damage. The favorite sport modalities among the active students are the individual sports without contact in the girls and the collective sport with contact between the males, the violent conducts were more frequent in the collective sports besides that the practicing students were exposed to situations of manifest victimization verbal and physical in most situations

Keywords: Victimization; Primary education; Sport; Schoolchildren

Tipe: Original - Section: Physical education

Author's number for correspondence: 1 - Sent: 10/01/2018; Accepted: 23/01/2018

${ }^{I}$ Departamento de Didáctica la Expresión Musical, Plástica y corporal, Universidad de Granada-España, felixzo@ugr.es

ORCID https://orcid.org/0000-0000-0000-0000

${ }^{2}$ Departamento de Educación Física, Facultad de Ciencias de la Educación, Universidad Internacional de La Rioja-España, manuel.castro@unir.net ORCID https://orcid.org/0000-0002-2357-3093

${ }^{3}$ Departamento de Didácticas Integradas, Facultad de Ciencias de la Educación, Universidad de Huelva, ramon.chacon@ddi.uhu.es, ORCID https://orcid.org/0000-0003-0937-1089,

Zurita Ortega, F.; Castro Sánchez, M.; Chacón Cuberos, R. (2018). Análisis de la influencia de la modalidad deportiva sobre la victimización en escolares de Primaria. ESHPA - Education, Sport, Health and Physical Activity 2(1): 2-15. doi: http://hdl.handle.net/10481/48262

ESHPA - Education, Sport, Health and Physical Activity - ISSN: 2603-6789 


\section{(P) Análise da influência da modalidade esportiva sobre a vitimização em crianças do ensino}

\section{fundamental}

\section{Resumo}

Introdução: Introdução: a violência e sua erradicação são um elemento que deve ser considerado de vital importância na educação, uma vez que os eventos de bullying ocorrem cada vez mais em uma idade precoce. A atividade física é um dos principais elementos para o trabalho de prevenção.

Objectivos: propõe-se este estudo com os objetivos de descrever os níveis de vitimização, atividade física e tipo de esporte em uma população de 9 a 12 anos da cidade de Granada e estabelecer a relação entre a prática eo tipo de atividade física e os níveis de vitimização.

Métodos: um total de 177 escolares de 10 a 12 anos $(\mathrm{M}=11,04$ anos, $\mathrm{SD}=1,258)$ da cidade de Granada participaram de um questionário Ad-Hoc e do questionário de vitimização na escola.

Conclusões: Resultados e discussão: os resultados mostraram que os três quartos dos alunos realizam atividades físicas nãoescolares, sendo isso mais freqüente entre os homens, caracterizando-se por uma maior taxa de vitimização física, enquanto o gênero feminino é caracterizado por maior vitimização Relacional, isto é, eles tendem a excluir pares e, embora isso seja menos visível, pode causar grandes danos psicológicos. As modalidades desportivas favoritas entre os alunos ativos são os esportes individuais sem contato nas raparigas e no esporte coletivo com contato entre os machos, as condutas violentas foram mais freqüentes no esporte coletivo, além de que os estudantes praticantes foram expostos a situações de vitimização manifesta verbal e físico na maioria das situações

Palavras-chave: (1-5). Vitimização; Educação primária; Esportes; Escolares 


\section{Introducción}

Se debe partir de la contrariedad tan grave que está aconteciendo en la sociedad actual con el problema de los comportamientos violentos en edades escolares como señalan estudios recientes de todo el mundo (Gaete y Araya, 2017; Jenkins y Nickerson, 2017; Myers y Bhopal, 2017; Wood, Smith, Varjas y Meyers, 2017), ante este hecho y previo a estos trabajos en España se elaboró el II Plan Estratégico Nacional de Infancia y Adolescencia 2013-2016, para personas menores de 18 años (Vilches, 2015), donde se trataban cuestiones tan elementales como la agresión escolar y la victimización, y de cómo poder intentar erradicar, minimizar y en la medida de lo posible controlar este fenómeno dentro de las escuelas. Algunos autores como Zurita, Vilches, Cachón, Padial, Martínez y Castro (2015), indican que la agresión se encuentra conformada por una serie de elementos que generan daño de tipo físico, moral o psicológico, que un individuo provoca a otro, no hay otra finalidad, que un beneficio para el agresor (Ramírez y Andreu, 2003), y que el agredido lo es en contra de su voluntad.

Para comprender las formas en que se presenta las conductas y comportamientos violentos debemos partir de las diversas clasificaciones propuestas teniendo en cuenta los modelos propuestos anteriormente; de esta manera Little, Henrich, Jones y Hawley (2003), hablan de cómo se ejerce la violencia tanto por su naturaleza (física o manifiesta, verbal y social) como por la relación interpersonal o Parrot y Giancola (2007) y Kuppens, Grietens, Onghena, Michiels y Subramanian (2008) en función de si hablamos de violencia directa-abierta o indirecta-relacional.

En la etapa de Educación Primaria la violencia que más aparece es la física, y este dato se invierte en la adolescencia donde la más usual es la violencia relacional (Moreno, Estévez, Murgui y Musitu, 2009) siendo complicada su detección debido a la sutileza empleada y a la falta de señales visuales, no es como las formas física y verbal, que se aprecian y manifiestan de manera bien visible.

Otro elemento que se debe tener en cuenta es que no todas las víctimas actúan de una forma similar, algunas de ellas actúan como personas muy tímidas o sumisas, y otras muy desafiantes, y a la larga se convierten en futuros agresores (Liebert y Birnes, 2010), con unos caracteres muy específicos, como son la escasez de habilidades sociales, impetuosos y con situaciones familiares hostiles.

En los últimos años ha aparecido una nueva modalidad de agresividad que es conocida como Bullying, este concepto viene determinado por una serie de causas o factores como son la familia, la escuela, los niveles de popularidad entre iguales y en tiempo dedicado a actividades audiovisuales, hoy en día se atienden a parámetros de tipo personal (Zurita y Álvaro, 2014), familiar (Serrano y Iborra, 2005), escolar (Calvo y Ballester, 2007; Choi y Calero, 2012), y social (Buelga, Musitu, Murgui y Pons, 2008).

Los factores asociados a las conductas y comportamiento violentos dentro del ámbito de los jóvenes es analizado y estudiado por numerosos científicos (Valdés, Estévez y Valenzuela, 2014; Durán, 2015; 
Cerezo, 2015; Calleja, 2016; Schuch y Munhoz, 2016), y entre ellos plantean un modelo que podría determinar los factores que inciden en la generación de comportamientos y conductas violentas.

El hecho de que las escuelas y centros educativos aparezcan en los medios de información de una manera habitual preocupa seriamente a todos los miembros de la comunidad educativa, puesto que son situaciones que causan una gran alarma social y que han generado multitud de denuncias en los juzgados a nivel nacional comunitario o internacional. Ante semejante situación, investigadores como Anderson y Bushman (2002), Garfiels y Llanten (2004), Correia, Vala, y Aguiar (2007) y Estévez, Inglés, Emler, MartínezMonteagudo y Torregrosa (2012), señalan que las cifras constituyen una epidemia de este siglo.

Si se analizan los comportamientos y conductas violentas por género algunos autores como Cabrera (2002), Garaigordobil, Álvarez y Carralero (2004), Ortega y Monk (2005), Loukas et al. (2005) o Toldos (2005), indican que es más común en los sujetos masculinos, y es la de tipo físico la que prevale, mientras que en los sujetos femenino la que se produce con mayor asiduidad es la de tipo relacional (Del Barrio, Martín, Montero, Gutiérrez y Fernández, 2003). La edad es otro elemento a tener en cuenta ya que durante las etapas más jóvenes se produce un mayor nivel de agresiones físicas cambiándose la tendencia al llegar a edades más adultas (Moffit y Caspi, 2001; Blaya, Debarbieux, Del Rey y Ortega, 2006).

El concepto deporte proviene de la palabra anglosajona "sport" y en su origen tiene una connotación eminentemente lúdica y de tipo recreativa. Algunos estudios resaltan la importancia del papel de la actividad física durante la infancia como un factor que está vinculado con beneficios para la salud física, conductual y psicológica, contribuyendo a la formación integral de los alumnos. Se destacan que algunos valores relacionados a la práctica deportiva como la amistad, autoestima, civismo, cooperación o competitividad (pero desde el punto de vista de la autosuperación y no de la rivalidad), y Monjas, Ponce y Gea (2015) añaden la necesidad de impulsar la deportividad para poder abordar la lucha contra la violencia escolar e impulsar la inclusión de todos los alumnos.

Como señalaba en su trabajo Zurita et al. (2015), la actividad física y por ende el deporte tiene como objetivos reducir e intentar prevenir el bullying y los índices de agresividad y violencia para ello se plantea que en los deportes de equipo el líder sea el encargado de controlar las relaciones y si se presenta algún problema solucionarlo, produciendo por parte de los compañeros empatía hacia los que pudiesen sufrir victimización. Este estudio nos aporta datos novedosos sobre los niveles de violencia en la etapa de Primaria, y se plantea con los objetivos de describir los niveles de victimización, actividad física y tipo de deporte en una población de 9 a 12 años de la ciudad de Granada y establecer la relación entre la práctica y tipología de actividad física y los niveles de victimización.

\section{Material y métodos}

\section{Diseño y Participantes}

Zurita Ortega, F.; Castro Sánchez, M.; Chacón Cuberos, R. (2018). Análisis de la influencia de la modalidad deportiva sobre la victimización en escolares de Primaria. ESHPA - Education, Sport, Health and Physical Activity 2(1): 2-15. doi: http://hdl.handle.net/10481/48262

ESHPA - Education, Sport, Health and Physical Activity - ISSN: 2603-6789 
Participaron en esta investigación de carácter descriptivo y de tipo transversal un total de 177 escolares de ambos sexos (59,9\% masculinos y 40,1\% femeninos), de 9 a 12 años (M=11,04 años; DT= 1,258), pertenecientes a centros ubicados en distintas zonas de la ciudad de Granada todos ellos distribuidos en Educación Primaria.

Variables e instrumentos

La presente investigación tomó como referencia las siguientes variables e instrumentos de medida, es decir:

- Edad, de 9 a 12 años.

- Género, Masculino o femenino.

- Realización de actividad física fuera del contexto escolar al menos tres horas a la semana, estructurado en sí o no

- Tipo de Deporte, Categorizado en individual sin contacto, individual con contacto, colectivo sin contacto y colectivo con contacto.

- Tipo de victimización, determinado en relacional, física o verbal., y extraído de la Escala de Victimización en la Escuela, adaptada al español por Cava, Musitu y Murgui (2007), donde mediante una escala Likert de cuatro opciones (1= Nunca a 4= Muchas veces), se valoran 20 ítems, que generan tres tipos de victimización en la escuela (Relacional, Física y Verbal). La consistencia interna (alfa de Cronbach) del cuestionario es de .924, $(\mathrm{p}=.888$ para Relacional, $\mathrm{p}=.568$ para Física y $\mathrm{p}=.846$ Verbal), datos similares a los hallados en el estudio de Povedano, Estévez, Martínez y Monreal (2012a). Para este estudio se siguen las aportaciones realizadas por Povedano et al., (2012a) que establece una medida general de victimización la cual resulta de sumar las puntuaciones de las tres subescalas de esta escala.

\section{Procedimiento}

En primer lugar, a través de la Facultad de Ciencias de la Educación de la Universidad de Granada (Área de Corporal), y en contacto con la Delegación de Educación de la Junta de Andalucía, se solicitó la colaboración de los Centros Educativos de la Capital de Granada seleccionados, a partir de un muestreo de conveniencia de las categorías objeto de estudio. La dirección de cada centro educativo fue informada sobre la naturaleza de la investigación y solicitando la colaboración de sus alumnos/as. En segundo lugar se adjuntó un modelo de autorización destinado a los responsables legales de los adolescentes pidiéndoles su consentimiento informado. En todo momento se garantizó a los participantes el anonimato de la información recogida aclarando que su utilización sería sólo con fines científicos. Los encuestadores estuvieron presentes durante la recogida de los datos que se llevó a cabo en sus aulas habituales durante un periodo regular de clase. La recogida se desarrolló sin ningún tipo de problema o anormalidad a reseñar. En último lugar se agradeció a los docentes, orientadores y responsables su colaboración y se les informó 
del envío en un futuro próximo de un informe sobre los datos obtenidos respetando la confidencialidad de los mismos.

El estudio cumplió con las normas éticas del Comité de Investigación y Declaración de Helsinki de 1975. Se contó en todos los casos con el consentimiento informado de los participantes y se respetó el resguardo a la confidencialidad.

\section{Análisis de los Datos}

Para el análisis de los datos se utilizó el programa estadístico software SPSS 23.0., mediante la utilización de diversas técnicas de análisis, de esta forma se establecieron los descriptivos básicos mediante la utilización de medias y frecuencias y para el estudio relacional se emplearon ANOVA y tablas de contingencia.

\section{Resultados}

En este estudio de 177 participantes el 79,7\% $(\mathrm{n}=141)$ de la muestra realizan actividad física más de tres horas semanales fuera del colegio frente al 20,3\% $(n=36)$ que no hacen, de ellos un $37,3 \%(n=66)$ realizan deporte de tipo colectivo con contacto, el 33,3\% $(n=59)$ lo desarrollan de tipo individual sin contacto, la modalidad individual con contacto es desarrollada por un $4 \%(n=7)$ mientras que el deporte colectivo sin contacto lo hacen un 5,1\% (n=9). En lo referente a los niveles de victimización se aprecia como la de tipo verbal $(M=2,71)$ es la que obtiene puntuaciones más altas, seguida de la relacional $(M=2,58)$, y por último la física $(\mathrm{M}=2,13)$, como se desprende de los datos de la siguiente tabla.

\begin{tabular}{|c|c|c|c|}
\hline \multicolumn{2}{|c|}{ Género } & \multicolumn{2}{|c|}{ Edad } \\
\hline Masculino & $59,9 \%(n=106)$ & 10 años & $32,7 \%(\mathrm{n}=58)$ \\
\hline Femenino & $40,1 \%(n=71)$ & 11 años & $49,2 \%(n=87)$ \\
\hline \multicolumn{2}{|c|}{ Actividad Física fuera escuela } & 12 años & $18,1 \%(n=32)$ \\
\hline No & $20,3 \%(\mathrm{n}=35)$ & \multicolumn{2}{|c|}{ Tipología de deporte } \\
\hline $\mathbf{S i}$ & $79,7 \%(n=142)$ & Individual Sin Contacto & $33,3 \%(n=59)$ \\
\hline \multicolumn{2}{|c|}{ Victimización } & Individual con Contacto & $4,0 \%(n=7)$ \\
\hline Victimización Verbal & $\mathrm{M}=2,71 ; \mathrm{DT}=1,163$ & Colectivo sin Contacto & $5,1 \%(\mathrm{n}=9)$ \\
\hline Victimización Física & $\mathrm{M}=2,13 ; \mathrm{DT}=1,088$ & Colectivo con Contacto & $37,3 \%(n=66)$ \\
\hline Victimización Relacional & $\mathrm{M}=2,58 ; \mathrm{DT}=1,087$ & No Practican & $20,3 \%(n=36)$ \\
\hline
\end{tabular}

Los resultados arrojaron la no aparición de diferencias estadísticamente significativas al comparar la variables de tipología deportiva y victimización según la edad $(\mathrm{p} \leq 0,05)$, donde si surgen es entre el género y la modalidad $(\mathrm{p}=0,000)$, generadas por un mayor porcentaje de práctica en el deporte colectivo con contacto en el sexo masculino 52,8\% $(\mathrm{n}=56)$ frente al 14,1\% $(\mathrm{n}=10)$ del femenino, inversamente es lo que sucede en el deporte individual sin contacto con predominio en el sexo femenino con $53,5 \%(n=38)$ frente al $19,8 \%$ del sexo masculino $(n=21)$. 


\begin{tabular}{|c|c|c|c|c|c|c|c|}
\hline & & \multicolumn{5}{|c|}{ Modalidad Deportiva } & \multirow[b]{2}{*}{ TOTAL } \\
\hline \multicolumn{2}{|c|}{ Género } & $\begin{array}{c}\text { Individual sin } \\
\text { Contacto }\end{array}$ & $\begin{array}{c}\text { Individual } \\
\text { con contacto }\end{array}$ & $\begin{array}{c}\text { Colectivo sin } \\
\text { contacto }\end{array}$ & $\begin{array}{c}\text { Colectivo con } \\
\text { contacto }\end{array}$ & $\begin{array}{c}\text { No } \\
\text { practica }\end{array}$ & \\
\hline \multirow{3}{*}{ Masculino } & Recuento & 21 & 7 & 6 & 56 & 16 & 106 \\
\hline & $\%$ Sexo & $19,8 \%$ & $6,6 \%$ & $5,7 \%$ & $52,8 \%$ & $15,1 \%$ & $100,0 \%$ \\
\hline & \% Deporte & $35,6 \%$ & $100,0 \%$ & $66,7 \%$ & $84,8 \%$ & $44,4 \%$ & $59,9 \%$ \\
\hline \multirow{3}{*}{ Femenino } & Recuento & 38 & 0 & 3 & 10 & 20 & 71 \\
\hline & $\%$ Sexo & $53,5 \%$ & $0,0 \%$ & $4,2 \%$ & $14,1 \%$ & $28,2 \%$ & $100,0 \%$ \\
\hline & $\%$ Deporte & $64,4 \%$ & $0,0 \%$ & $33,3 \%$ & $15,2 \%$ & $55,6 \%$ & $40,1 \%$ \\
\hline \multirow{3}{*}{ Total } & Recuento & 59 & 7 & 9 & 66 & 36 & 177 \\
\hline & $\%$ Sexo & $33,3 \%$ & $4,0 \%$ & $5,1 \%$ & $37,3 \%$ & $20,3 \%$ & $100,0 \%$ \\
\hline & \% Deporte & $100,0 \%$ & $100,0 \%$ & $100,0 \%$ & $100,0 \%$ & $100,0 \%$ & $100,0 \%$ \\
\hline
\end{tabular}

También se encuentran al relacionar el sexo de los participantes con los niveles de victimización, diferencias estadísticas significativas $(\mathrm{p} \leq 0,050)$, encontrando que en la victimización física, los chicos obtienen cifras superiores $(M=2.35)$ que las chicas $(M=1,80)$, respecto a la victimización verbal sucede igual que antes y los chicos obtienen cifras superiores $(M=2,80)$ y las chicas $(M=2,53)$ mientras que en la victimización relacional esta tendencia se invierte, siendo las cifras de las chicas superiores $(\mathrm{M}=2,63)$ a los chicos $(M=2,54)$, como se aprecia en la siguiente tabla.

\begin{tabular}{|c|c|c|c|c|c|}
\hline \multicolumn{2}{|c|}{ Sexo } & Media & Desviación Típica & $\mathbf{F}$ & $\mathbf{P}$ \\
\hline \multirow{2}{*}{ Victimización Física } & Masculino & 2,35 & 1,163 & \multirow[b]{2}{*}{12,137} & \multirow[b]{2}{*}{0,001} \\
\hline & Femenino & 1,80 &, 874 & & \\
\hline \multirow{2}{*}{ Victimización Verbal } & Masculino & 2,80 & 1,114 & \multirow{2}{*}{6,143} & \multirow{2}{*}{0,000} \\
\hline & Femenino & 2,58 & 1,229 & & \\
\hline \multirow{2}{*}{ Victimización Relacional } & Masculino & 2,54 & 1,034 & \multirow{2}{*}{5,789} & \multirow{2}{*}{0,000} \\
\hline & Femenino & 2,63 & 1,167 & & \\
\hline \multicolumn{2}{|c|}{ Práctica Deportiva } & Media & Desviación Típica & $\mathbf{F}$ & $\mathbf{P}$ \\
\hline \multirow{5}{*}{ Victimización Física } & Individual sin contacto & 2,23 & 1,139 & \multirow{5}{*}{0,358} & \multirow{5}{*}{0,838} \\
\hline & Individual con contacto & 1,75 & ,968 & & \\
\hline & Colectivo sin contacto & 2,61 & 1,490 & & \\
\hline & Colectivo con contacto & 2,14 & 1,079 & & \\
\hline & No Práctica & 1,93 & ,907 & & \\
\hline \multirow{5}{*}{ Victimización Verbal } & Individual sin contacto & 2,80 & 1,203 & \multirow{5}{*}{0,967} & \multirow{5}{*}{0,427} \\
\hline & Individual con contacto & 2,40 & ,971 & & \\
\hline & Colectivo sin contacto & 3,12 & 1,168 & & \\
\hline & Colectivo con contacto & 2,78 & 1,141 & & \\
\hline & No Práctica & 2,39 & 1,152 & & \\
\hline \multirow{5}{*}{ Victimización Relacional } & Individual sin contacto & 2,78 & 1,184 & \multirow{5}{*}{1,192} & \multirow{5}{*}{0,316} \\
\hline & Individual con contacto & 1,91 & ,539 & & \\
\hline & Colectivo sin contacto & 2,77 & 1,080 & & \\
\hline & Colectivo con contacto & 2,50 & 1,024 & & \\
\hline & No Práctica & 2,46 & 1,075 & & \\
\hline
\end{tabular}

Como se desprende de la tabla anterior no se encontró asociación estadística entre la victimización de tipo verbal, física y relacional con la modalidad deportiva que practican. 


\section{Discusión}

En este trabajo de investigación realizado sobre 177 alumnos de entre los 10 y 12 años, se establecen relaciones con los estudios de Andreou, Didaskalou y Vlachou (2015), con respecto a la práctica de actividad física, obteniendo cifras similares al mismo, y concuerdan con las cifras de los estudios de Chacón, Zurita, Castro y Linares (2017) donde reportaban que ocho de cada diez encuestados entre 10 y 12 años realizan actividad física fuera del colegio, mientras que difieren de los estudios de Reverter, Plaza, Jové y Hernández (2014), realizados a una muestra de 1248 alumnos comprendidos entre 6 y 12 años en la ciudad de Torrevieja (Alicante) se han encontrado cifras diferentes representando la práctica de actividad física, con un $65,6 \%$ y un $34,4 \%$ cifras ligeramente inferiores entendemos que motivadas porque el rango de edad de estos escolares es superior y hacen disciplinas de mayor índole competitiva y en ocasiones estos clubes se encuentran alejado del domicilio familiar, por lo que los padres o algún familiar tienen que encargase de llevarlo a la actividad y en muchas situaciones no disponen del tiempo o interés.

A pesar de que la mayoría de alumnos realiza actividad física fuera del colegio se encuentran diferencias en función del sexo siendo los niños más activos que las niñas estos datos siguen la misma línea de los estudios de Díaz, Carrillo y Borrego (2015), por otra parte estos datos no coinciden con los estudios de Luengo (2007) ya que se encuentra una diferencia más amplia entre género, entendiéndose que la menor práctica se encuentra relacionada con factores biológicos (los hombres tienen mayor masa y fuerza por lo general, mientras que las mujeres tienen mayor flexibilidad) de esto que la mayoría de chicas se dediquen a modalidades distintas de deportes que los chicos, también el tamaño del corazón de las mujeres es más pequeño así como su rendimiento puede verse afectado por el ciclo menstrual.

El deporte de tipo colectivo con contacto es el más valorado y realizado por los participantes seguido de la modalidad deportiva individual sin contacto mientras que con una menor representación se encuentran el deporte colectivo con contacto por los deportes individuales sin contacto, si relacionamos estos datos relacionados con el sexo indican que el sexo femenino tiene preferencia por realizar deportes individuales sin contacto como la gimnasia rítmica, mientras que dentro del sexo masculino optan por la realización de los deportes colectivos con contacto, siendo el deporte estrella el futbol, este estudio concuerda con los resultados ofrecidos en el estudio de Luengo (2007). Atendiendo a los niveles de victimización, se aprecia como la de tipo verbal es la que mostró una tendencia media superior que las otras dos variantes, en el tercer ciclo de Educación Primaria seguida de la victimización relacional y por último la física estos datos se encuentran relacionados con el informe del Defensor del Pueblo (2007), el cual, indica que los últimos datos obtenidos en nuestros país coinciden con que la verbal es la que está más presente con un porcentaje que oscila entre el 55,8\% y el 49\%, seguida a gran distancia de la victimización relacional, mientras que el último lugar está representado por la victimización física. 
A pesar de la menor frecuencia de la victimización física. esta es más frecuente en el sexo masculino, por otro lado se produce de forma inversa en la victimización relacional en la que las chicas obtienen unos valores más altos, corroborados por los estudio de Zurita et al. (2015), de donde se puede deducir que en los alumnos predominan más los ataques físicos debido a la tendencia de considerar este sexo más violento, más fuerte así como las preferencias del sexo femenino la hora de elegir sus grupos siendo mayor la frecuencia de exclusión de compañeros así como la imposición de motes a los mismos; pero esto no indica que solo los niños realicen agresiones físicas, sino que las chicas tienden más a realizar este tipo de victimización de forma indirecta.

Los estudios realizados en otros lugares del mundo obtuvieron cifras similares al nuestro, como el GómezBahillo, Puyal, Elboj, Sanz y Sanagustin (2005) en Aragón. En su trabajo de investigación sobre 535 alumnos de 10 a 16 años, encontró cifras de víctimas en un 4,3\% y de agresores en un 5,2 \%; el de Pérez, Fuentes y Gázquez (2010) que sitúan sobre una población de 1.214 estudiantes de 12 a 16 años, a las víctimas en un 2,1\% y a los agresores en un 3,5\%, o el de Sáenz, Calvo, Fernández y Silvan (2005) en la Rioja sobre 1053 alumnos de 8 a 18 años donde se arrojaron cifras de un 3,6\% de víctimas frente a un 6,2\% de agresores.

Todos estos estudios anteriormente citados guardan cierta homogeneidad y consenso a la hora de considerar la violencia escolar como: "aquella conducta intencionada mediante la cual se causa un daño a otra persona dentro del centro educativo o durante el trascurso de cualquier actividad externa e interna organizada por este, pudiendo ser ejercida o padecida por cualquier miembro de la comunidad educativa" (Guerra, Álvarez-García, Dobarro, Núñez, Castro y Vargas 2011, p.76).

Más recientemente tenemos los estudios de diversos científicos a lo largo de todo el mundo que denota que es un problema de índole mundial y que son multifactoriales los conceptos que se desarrollan, Hong y Espelage (2012) analizaron un modelo relacionado con la victimización entre personas que estudian en el mismo centro, y en ese trabajo se analizan los factores de riesgo. De esta manera Wandera et al. (2017) en Uganda estudiaban que factores se encontraban asociados a la victimización en escolares concretamente en 3706, indicaban cifras en torno al 30\% de violencia; en España Álvarez-García, Núñez, Barreiro y García (2017) validaron un cuestionario relacionado con el ciberbullying en una muestra de escolares preadolescentes.

En Chile Gaete y Araya (2017), realizaron un estudio que intentaba relacionar el uso de sustancias nocivas con otros elementos en una muestra superior a los 45000 participantes, se hallaron asociaciones entre diversos elemento como tener más tiempo dedicado a los amigos o poco control de los padres y BanzonLibrojo, Garabiles y Peña (2017), en Filipinas analizaron a 401 estudiantes donde señalaban victimización por intimidación por parte del docente y en este caso existe una percepción negativa por parte del estudiante 
hacia el docente, por lo que se sugiere que estrategias de disciplinas y comportamientos aceptables por parte del docente ocasionará una disminución en las conductas de agresividad.

\section{Conclusiones}

Las conclusiones que se obtienen de este estudio indican que:

Tres cuartos de los escolares realizan actividad física no escolar, siendo esta más asidua entre los varones, y estos se caracterizaban por tener un índice superior de victimización física mientras que el género femenino se caracteriza por tener mayor victimización relacional, es decir tienden más a excluir a los compañeros y aunque esta sea menos visible puede provocar grandes daños psicológicos.

Las modalidades deportivas favoritas entre los alumnos activos son los deportes individuales sin contacto en las chicas y el deporte colectivo con contacto entre los varones, las conductas violentas eran más frecuentes en los deportes colectivos además de que los alumnos practicantes se exponían a situaciones de victimización manifiesta verbal y física en la mayoría de situaciones.

\section{Conflict of interests / Conflicto de intereses}

No hay ningún conflicto

\section{References / Referencias}

Álvarez-García, D., Núñez, J. C., Barreiro, A., y García, T. (2017). Validation of the Cybervictimizacion Questionnaire (CYVIC) for adolescents. Computers in Human Behavior, 70, 270-281.

Anderson, C.A y Bushman, B.J. (2002). Human aggression. Annual Review of Psychology, 53, $27-51$.

Andreou ,E., Didaskalou ,E y Vlachou, A .(2015). Bully/victim problems among Greek pupils with special educational needs: associations with loneliness and self-efficacy for peer interactions. Journal of Research in Special Educational Need, 15(4), 235-246.

Banzon-Librojo, L., Garabiles, M. R., y Peña, L. (2017). Brief report: relations between harsh discipline from teachers, perceived teacher support, and bullying victimización among high school students. Journal of Adolescence, 57, 18-22.

Blaya, C., Debarbieux, E., Del Rey, R. y Ortega, R. (2006). Clima y violencia escolar. Un estudio comparativo entre España y Francia. Revista de Educación, 339, 293-315.

Buelga, S., Musitu, G., Murgui, S. y Pons, J. (2008). Reputation and aggressive behavior in adolescence. The Spanish Journal of Psychology, 11, 192-200. 
Cabrera, O. (2002). Psychologycal and behavioral correlates of adolescent gang involment. Dissertattion Abstracts International: Section B. Sciences and Engineerin, 62(11), 5405.

Calleja, N. (2016). Bullying y tabaco: ¿Se encuentran asociados?. Acta de Investigación Psicológica, 6(1), $2350-2367$.

Calvo, A.R. y Ballester, F. (2007). Acoso escolar: procedimientos de intervención. Madrid: Eos.

Cava, M.J., Musitu, G., y Murgui, S. (2007). Individual and social risk factors related to overt victimization in a sample of Spanish adolescents. Psychological Reports, 101, 275-290.

Cerezo, F. (2015). Bullying homofóbico. El papel del profesorado. International Jounal of Development and educational Psychology. Revista INFAD de Psicología, 1(1), 417-424.

Chacón, R., Zurita, F., Castro, M y Linares, M. (2017). Relación entre práctica físico-deportiva y conductas violentas en escolares de Educación Primaria de la provincia de Granada. Revista TécnicoCientífica del Deporte Escolar Educación Física y Psicomotricidad, 3(1), 3-15.

Choi, A. y Calero, J. (2012). Rendimiento académico y titularidad de centro en España. Profesorado: Revista de currículum y formación del profesorado, 16(3), 31-57.

Correia, I., Vala, J., y Aguiar, P. (2007). Victim`s innocence, social, categorization, and the threat to the belief in a just world. Journal of Experimental Social Psychology, 43, 31-38.

Del Barrio, C., Martín, E., Montero, I., Gutiérrez, H. y Fernández, I. (2003). La realidad del maltrato entre iguales en los centros de secundaria. Infancia y Aprendizaje, 26(1), 25-47.

Díaz, A., Carrillo, A. y Borrego, F. (2015). Análisis descriptivo de la práctica físico-deportiva e intereses de práctica en escolares de $5^{\circ}$ y $6^{\circ}$ de primaria. Sport TK. 4(1), 23-28.

Durán, M. (2015). Ciberacoso mediante teléfono móvil e internet en las relaciones de noviazgo entre jóvenes. Comunicar, 22(44), 159-167.

Estévez, E., Inglés, C., Emler, N., Martínez-Monteagudo, M. C. y Torregrosa, M. S. (2012). Análisis de la relación entre la victimización y la violencia escolar: El rol de la reputación antisocial. Intervención Psicosocial, 21, 53-65.

Gaete, J. y Araya, R. (2017). Individual and contextual factors associated with tobacco, alcohol and cannabis use among Chilean adolescents: a multilevel study. Journal of Adolescence, 56, 166-178.

Garaigordobil, M., Álvarez, Z. y Carralero, V. (2004). Conducta antisocial en niños de 10 a 12 años: Factores de personalidad asociados y variables predictoras. Análisis y Modificación de Conducta, 130, 241-271

Garfield, R. y Llanten C. (2004). The public health context of violence in Colombia. Revista Panamericana Salud Publica, 16(4), 266-271.

Gómez-Bahillo, C., Puyal, E., Elboj, C., Sanz, A. y Sanagustín, M. V. (2005). Comportamiento social de los estudiantes de educación no universitaria en la comunidad aragonesa. Estudio preliminar. 
Primera parte. El conflicto relacional en el ámbito educativo. Extraído el 23 de abril del 2012 desde: http://bases.cortesaragon.es/bases/NDocumen.nsf/9cd39ed0285dc9b2c12570a1002a3f65/97f4488 6f93f372ec125758d0039eee7/\$FILE/indice_INFORME\%20PRELIMINAR.pdf

Guerra, C., Álvarez-García, D., Dobarro, A., Núñez, J. C., Castro, L. y Vargas, J. (2011). Violencia escolar en estudiantes de Educación Secundaria de Valparaíso (Chile): Comparación con una muestra española. Revista Iberoamericana de Psicología y Salud, 2, 75-98.

Hong, J. S. y Espelage, D. L. (2012). A review of research on bullying and peer victimización in school: an ecological system analysis, Aggression and Violent Behavior, 17(4), 311-322.

Jenkins, L. y Nickerson, A. (2017). Bullying participants roles and gender as predictors of bystander intervention. Aggressive Behavior, 43(3), 281-290.

Kuppens, S., Grietens, H., Onghena, P., Michiels, D. y Subramanian, S. V. (2008). Individual and classroom variables associated with relational aggression in elementary-school aged children: A multilevel analysis. Journal of School Psychology, 46, 639-660.

Liebert, J. y Birnes, W. (2010). Asesinos en Masa suicidas: Un estudio criminológico acerca del por qué matan. Estados Unidos: CRC Press.

Little, T. D., Henrich, C. C., Jones, S. M., y Hawley, P. H. (2003). Disentangling the "whys" from the "whats" of aggressive behaviour. International Journal of Behavioral Development, 27, 122-133.

Loukas, A., Paulos, S.K., y Robinson, S. (2005). Early adolescent social and overt aggression: Examining the roles of social anxiety and maternal psychological control, Journal of Youth and Adolescence, $34,335-345$.

Luengo, C. (2007). Actividad físico-deportiva extraescolar en alumnos de primaria. Revista Internacional de Medicina y Ciencias de la Actividad Física y el Deporte, 7(27), 174-184.

Moffitt, T. E. y Caspi, A. (2001). Childhood predictors differentiate life-course persistent and adolescence-limited antisocial pathways among males and females, Development and Psychopathology, 13, 355-375.

Monjas, R., Ponce, A. y Gea, J. M. (2015). La transmisión de valores a través del deporte. Deporte escolar y deporte federado: relaciones, puentes y posibles transferencias. Retos, 28, 276-284.

Moreno, D., Estévez, E., Murgui, S. y Musitu, G. (2009). Reputación social y violencia relacional en adolescentes: el rol de la soledad, la autoestima y la satisfacción vital. Psicothema, 21, 537-542.

Myers, M. y Bhopal, K. (2017). Racism and bullying in rural primary schools: protecting white identities post Macpherson. British Journal of Sociology of Education, 38(2), 125-143.

Ortega, R. y Monk, C. (2005). Agresividad injustificada entre preescolares. Psicothema, 17(3), 453-458. 
Parrott, D.J. y Giancola, P.R. (2007). Addressing "the criterion problema" in the asessment of aggressive behavior: development of a new taxonomic system. Aggression and Violent Behavior, 12, 280 299.

Pérez-Fuentes, M.C. y Gázquez, J.J. (2010). Variables relacionadas con la conducta violenta en la escuela según los estudiantes. International Journal of Psychology and Psychological Therapy, 10, 427437.

Povedano, A., Estévez, E., Martínez, B., y Monreal, M.C. (2012). Un perfil psicosocial de adolescentes agresores y víctimas en la escuela: análisis de las diferencias de género. Revista de Psicología Social, 27 (2), 169-182.

Ramírez, J. M. y Andreu, J. M. (2003). Agression's typologies. International Review of Social Psychology, $16,125-141$.

Reverter, J., Plaza, D., Jové, M. C., y Hernández, V. (2014). Actividad física extraescolar en alumnos de Primaria: el caso de Torrevieja (Alicante).Retos, 25,28-42.

Sáenz, T., Calvo, J., Fernández, F. y Silván, A. (2005) El acoso escolar en los centros educativos de La Rioja. La Rioja: Informe inédito del Servicio de Inspección Técnica Educativa. Sector Rioja Baja - Logroño Este.

Schuch, A. y Munhoz, T. N. (2016). Victimizacao por bullying em estudantes: estudo transversal. Adolescencia e Saude, 13(3), 7-15.

Serrano, A. y Iborra, I. (2005). Violencia entre compañeros en la escuela. Valencia: Centro Reina Sofía para el estudio la violencia.

Toldos, M.P. (2005). Sex an age difference in self-estimated physical, verbal and indirect agressive in Spanish adolescent. Aggressive Behavior, 31, 13-23.

Valdés, A. A., Estévez, E. H., y Valenzuela, A. M. (2014). Creencias de docentes acerca del bullying. Perfiles educativos, 36(145), 51- 64.

Vílches, J. M. (2015). Centros especializados y normalizados de secundaria: relación entre autoestima, agresividad, victimización y calidad de vida en estudiantes de granada capital. Tesis Doctoral: Universidad de Granada.

Wandera, S. O., Clarke, K., Knight, L., Allen, E., Walakira, E., Namy, S., Naker, D., y Devries, K. (2017). Violence against children perpetrated by peers: A cross-sectional school-based survey in Uganda. Child Abuse \& Neglect, 68, 65-73.

Wood, L., Smith, J., Varjas, K., y Meyers, J. (2017). School personnel social support and nonsupport for bystanders of bullying: exploring student perspectives. Journal of School Psychology, 61, 1-17. 
Zurita, F., Vilches, J. M., Cachón, J., Padial, R., Martínez, A., y Castro, M. (2015). Violencia escolar en adolescentes: un análisis en función de la actividad física y lugar de residencia habitual. Universitas Psychologica, 14(2), 743-754.

Zurita, F., y Álvaro, J. I. (2014). Repercusión del tabaco y el alcohol sobre factores académicos y familiares en adolescentes. Health and Addictions, 14(1), 59-70. 